\title{
A Wide-Band 2-Path Cross-Coupled Sigma Delta ADC
}

\author{
Erkan Bilhan \\ Texas Instruments Inc. \\ App. Spec. Prod. Group \\ Dallas, TX, USA \\ ebilhan@ti.com
}

\author{
Franco Maloberti \\ Department of Electronics \\ University of Pavia \\ Pavia, Italy \\ franco.maloberti@unipv.it
}

\begin{abstract}
The performance of a sigma-delta ADC can be increased by increasing one or more of the main three parameters, over-sampling ratio, the order of the modulators and the number of bits used. Increasing each of these parameters presents a degree of challenge (i.e., the increase in the over-sampling ratio is limited by the technology and the power consumption requirement). This paper presents a new method to obtain $2^{\text {nd }}$ order noise shaping from two $1^{\text {st }}$ order, time interleaved modulators by applying cross-coupling of quantization noise between the two paths. The proposed sigmadelta ADC is implemented in 90nm CMOS technology.
\end{abstract}

\section{INTRODUCTION}

The portable semiconductor market demands high performance analog-to-digital converters (ADCs) in terms of resolution and signal bandwidth with stringent requirements on the power consumption, in order to maintain a longer battery life. Also, the analog circuitries are required to be integrated with the digital circuitries to provide system-onchip solutions (SOCs). This leads to a need for analog circuitries to be implemented in digital technologies where analog friendly, high performance and precision devices do not exist. For the portable applications sigma-delta ADCs are preferred due to their high performance in the absence of precision components, and their low power consumption compared to their counterpart Nyquist rate converters.

The resolution of a sigma-delta ADC can be increased by increasing one or more of the three parameters; number of bits used for the flash, over-sampling ratio (OSR), and the order of the modulator [1]. The increase in the number of bits is limited due to the exponential increase in the area consumption as well as the complexity of the implementation of the digital-toanalog converter (DAC) in the feedback loop. OSR can be increased by increasing the sampling frequency; however, this increases the power consumption of the analog circuits. Higher order of modulators can be achieved by either cascading low order modulators or increasing the number of integration loops in single loop architectures. Beyond $2^{\text {nd }}$ order, the single loop modulators exhibit stability problems. Due to this apparent trade-off, the system architecture and parameters have to be optimized to meet the desired system specifications.

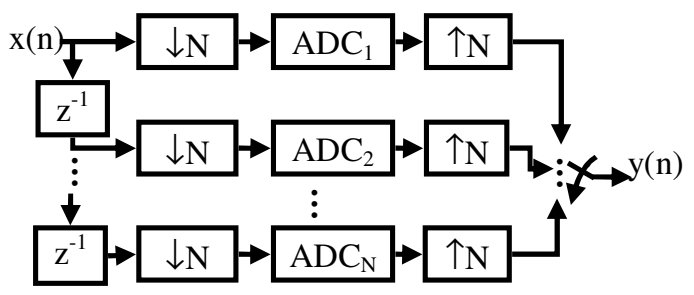

Fig. 1. N-path ADC.

This paper presents a new sigma-delta modulator architecture that utilizes $1^{\text {st }}$ order, two-path, time interleaved modulator to obtain a $2^{\text {nd }}$ order noise shaping. The increase in the order of the modulator is achieved by cross- coupling of the quantization noise between two paths. The modulator architecture has the advantage of reducing the speed of the conversion by half, compared to traditional $2^{\text {nd }}$ order modulators. Therefore, it enables low power implementations for low OSR, wide bandwidth applications. Furthermore, the proposed $2^{\text {nd }}$ order modulator can be implemented with just one opamp. The reduction in active components enables further power savings. The sigma delta ADC is implemented and simulated using a 90nm CMOS technology.

Next section of the paper reviews the time-interleaved architectures and introduces the proposed cross-coupling twopath sigma delta ADC architecture. The system level verification of the proposed architecture via Simulink simulations is reported in Section III. Then, Section IV presents the circuit implementation and Spice simulation results. Finally, Section V summarizes the conclusions.

\section{THE PROPOSED 2-PATH SigMA-DELTA ADC ARCHITECTURE}

\section{A. Review of the multi-path ADC architectures}

Multi-Path converters employ parallelism to increase the throughput of the conversion system. For this purpose arrays of converters are used in parallel [2], see Fig. 1. The effective rate of conversion increases by the number of parallel paths, which is equivalent to increasing the OSR of a single stage by the number of parallel paths. If sigma-delta modulators are 


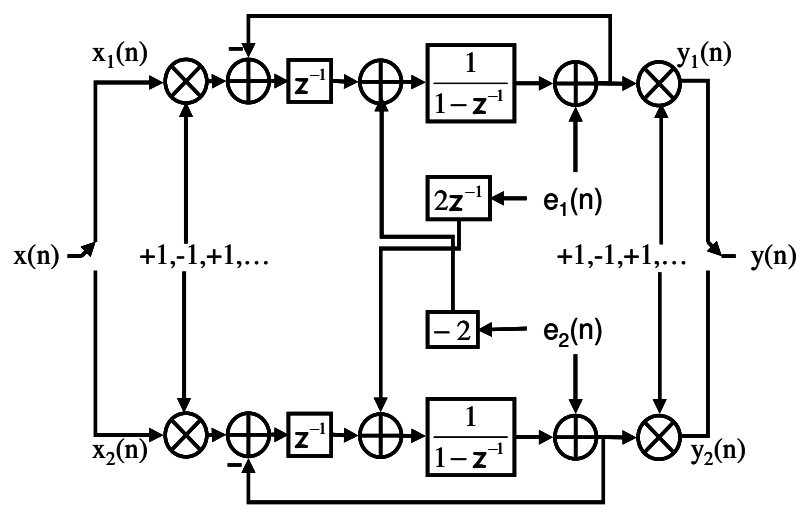

Fig. 2. The proposed sigma delta ADC architecture

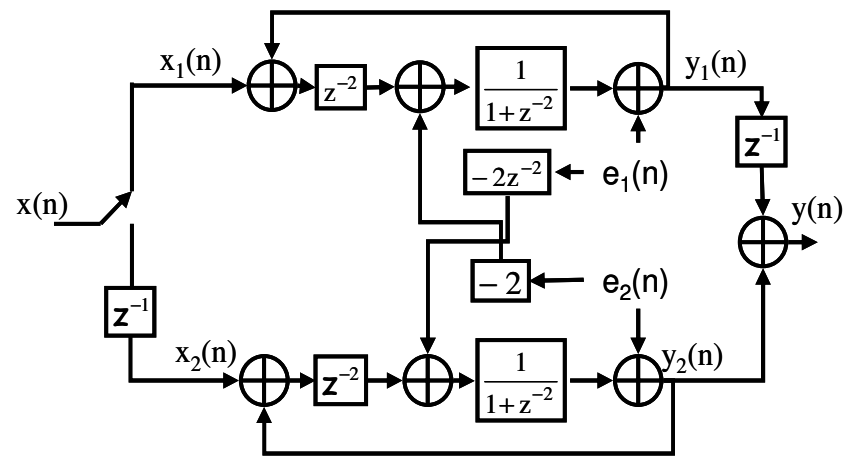

Fig. 3. The proposed modulator after $\mathrm{z} \rightarrow-\mathrm{z}^{2}$.

simply placed in parallel to build multi-path architectures, overall improvement in the resolution is only $3 \mathrm{~dB}$ for each doubling of the number of the paths, regardless of the order of the modulators used in the multi-paths. The improvement is due to the increase in the output data rate with the increase in the number of paths. The overall quantization noise of the system is not shaped. However, there are several reported multi-path sigma-delta modulator architectures that maintain the noise shaping characteristics as well, [3]-[17]. These architectures can be summarized under three groups:

- Multi-band sigma delta modulators are composed of parallel modulators that reject the quantization noise at a certain frequency band. The quantization noise out of the each band of each path is filtered out to allow reconstruction of the signal [3], [4].

- Modulation based sigma delta modulators modulate and demodulate the input and the output of each path, respectively, with a certain function, [5]-[9]. The output of each path is then filtered to generate the digitized signal.

- Time-interleaving sigma-delta modulators that apply block filtering principles to mutually cross-couple the paths [10]-[17].

The performances of all of these three groups are similar but the time-interleaving architectures offer area efficient implementations [12].

\section{B. Proposed cross-coupled sigma delta ADC}

The proposed sigma-delta ADC architecture is composed of two time-interleaved low-pass sigma delta modulators, as shown in Fig. 2 . The quantizers are represented by their linear models and the quantization noises of the each path are crosscoupled with a gain of two between these two modulators. The interleaved paths operate at half of the output data rate, $\mathrm{F}_{\mathrm{S}}$ and the input signal is sub-sampled in alternating polarities in order to modulate the sampled signal with \pm 1 pulse train. Therefore, if it is referred back to the highest clock frequency, this is equivalent to applying $\mathrm{z} \rightarrow-\mathrm{z}^{2}$ transform to the system [18], [19]. The system architecture after $z \rightarrow-z^{2}$ transform is depicted in Fig. 3. The negative sign that appears for the input after the transformation is arbitrary, hence it is ignored.

The transfer function of the system can be obtained by writing the outputs of each path, $\mathrm{y}_{1}(\mathrm{n})$ and $\mathrm{y}_{2}(\mathrm{n})$ in $\mathrm{z}$-domain as below

$$
\begin{aligned}
& Y_{1}(z)=z^{-2} X_{1}(z)-2 E_{2}(z)+\left(1+z^{-2}\right) E_{1}(z) \\
& Y_{2}(z)=z^{-2} X_{2}(z)-2 z^{-2} E_{1}(z)+\left(1+z^{-2}\right) E_{2}(z)
\end{aligned}
$$

The input, $\mathrm{X}(\mathrm{z})$ and the output $\mathrm{Y}(\mathrm{z})$ of the system are equal to the summations of the input and the output of the two paths, respectively:

$$
\begin{aligned}
& X(z)=z^{-1} X_{1}(z)+X_{2}(z) \\
& Y(z)=z^{-1} Y_{1}(z)+Y_{2}(z)
\end{aligned}
$$

Also, we can define a quantization noise, e(n) for the overall system as

$$
E(z)=z^{-1} E_{1}(z)+E_{2}(z)
$$

Substituting (1), in (3) and rearranging according to (2) and (4) yield the systems transfer function

$$
Y(z)=z^{-2} X(z)+\left(1-z^{-1}\right)^{2} E(z) .
$$

The system equation in (5) reveals that by cross-coupling the quantization noises of the two paths with a gain of -2 , a low pass sigma-delta modulator with a $2^{\text {nd }}$ order noise shaping is obtained from $1^{\text {st }}$ order time-interleaved modulators. The proposed architecture offers $15 \mathrm{~dB}$ improvement in the SNR of the sigma delta modulator per each doubling of the OSR, while a traditional two-path modulator built with the same $1^{\text {st }}$ order modulators can offer only $9 \mathrm{~dB}$ improvement per each doubling of the OSR. Also, compared to a traditional second order sigma delta modulator, the proposed architecture has the advantage of having the analog circuits operate at the half of the output data rate while providing the same performance. As a result of this, the constraints on the analog circuitries are relaxed. Besides, traditional $2^{\text {nd }}$ order sigma-delta modulator requires two integrators. But, with the proposed architecture, the number of integrators can be reduced by sharing one integrator between the two paths. Consequently, the power and area consumption are improved. 


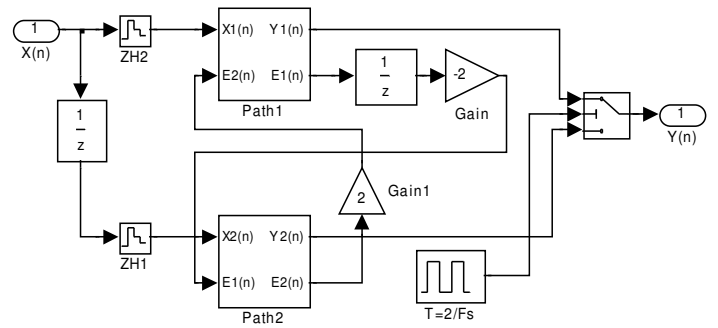

(a)

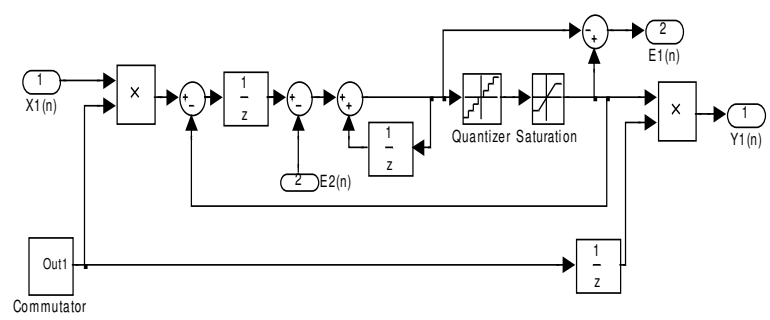

(b)

Fig. 4. Simulink model of (a) the top level and (b) the modulator architecture of each path.

TABLE I. The Simulink Model Parameters

\begin{tabular}{|l|c|}
\hline Signal Bandwidth & $2 \mathrm{MHz}$ \\
\hline Output Data Rate & $60 \mathrm{MHz}$ \\
\hline OSR & 15 \\
\hline Input Signal Frequency & $622 \mathrm{KHz}$ \\
\hline
\end{tabular}

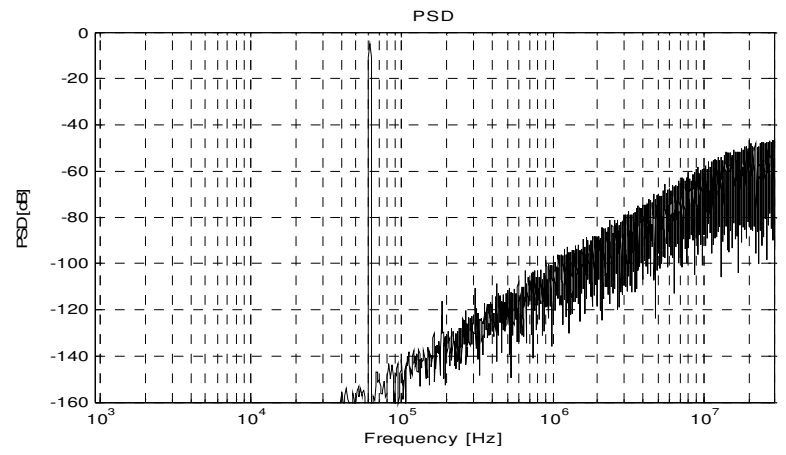

Fig. 5. The power spectral density of the Simulink model with $-1 \mathrm{~dB}$ input.

\section{SYSTEM LEVEL SiMULATIONS WITH SIMULINK}

In order to verify the proposed sigma-delta modulator architecture, a Simulink model is developed, see Fig. 4. The quantization noises are cross-coupled with a gain of two and a negative polarity is used for the first channel, as shown in Fig. 4(a). The modulators in each path are $1^{\text {st }}$ order low-pass sigma delta ADCs with nine level quantizers, Fig. 4(b). The commutator in the model modulate the input signal with sequence of $\{+1,-1,+1, \ldots\}$. The commutator can be eliminated by simply applying the $\mathrm{z} \rightarrow-\mathrm{z}^{2}$ transform to the modulator model. But, it is kept just because the model is built to closely represent all the elements of the actual circuit implementation of the modulator. Also, a $2^{\text {nd }}$ order sigma-
TABLE II. Simulink SimUlation SNDR REsults For Proposed ARCHITECTURE AND THE REFERENCE SYSTEM

\begin{tabular}{|l|c|c|}
\hline $\begin{array}{c}\text { Input Signal } \\
\text { Level [dB] }\end{array}$ & $\begin{array}{c}\text { Proposed } \\
\text { Modulator } \\
{[\text { dB] }}\end{array}$ & $\begin{array}{c}2^{\text {nd }} \text { Order } \\
\text { Reference } \\
{[\text { dB] }}\end{array}$ \\
\hline-48 & 13 & 17 \\
\hline-24 & 39 & 42 \\
\hline-12 & 54 & 55 \\
\hline-6 & 59 & 59.6 \\
\hline-3 & 62 & 60 \\
\hline
\end{tabular}

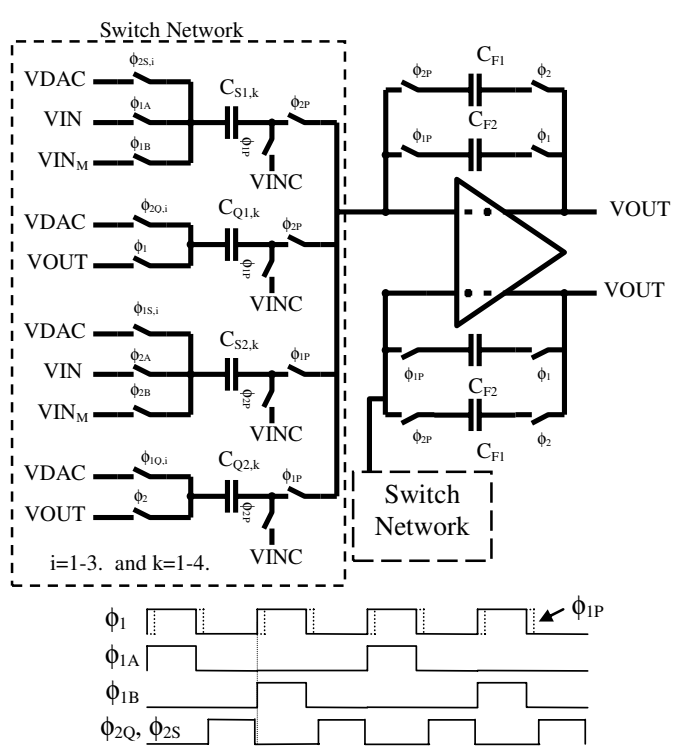

Fig. 6. The implementation of the integrator for both of the paths.

delta modulator model is used as a reference system to benchmark the results obtained. The parameters used for the Simulink simulations for both of the models are summarized in Table I.

The Simulink simulations of the proposed modulator show exactly the same spectral density for the quantization noise as the $2^{\text {nd }}$ order sigma delta modulator (see Fig. 5). This result is also inline with the theoretical result given in (5). The Simulink simulations were also performed with analog circuit non-idealities such as finite amplifier gain, gain-bandwidth and slew-rate [20]. The SNDR performance of proposed modulator closely follows the reference modulator's performance for signals with input levels higher than $-12 \mathrm{~dB}$ as shown in Table II. But the SNDR of proposed modulator degrades more than the SNDR of reference modulator for input levels lower than $-12 \mathrm{~dB}$.

\section{CIRCUIT IMPLEMENTATION}

The $1^{\text {st }}$ order sigma delta ADCs used in each path is composed of an integrator, a quantizer and a feedback DAC. Since, the integrator and the quantizer of each path are active during only one phase of the clock, the opamp of the integrator and the quantizer are shared between the two paths. But, two separate sets of capacitors, one for each path, are used to implement the integrator and the DAC as shown in Fig. 6. The DAC is implemented using switched capacitors. 


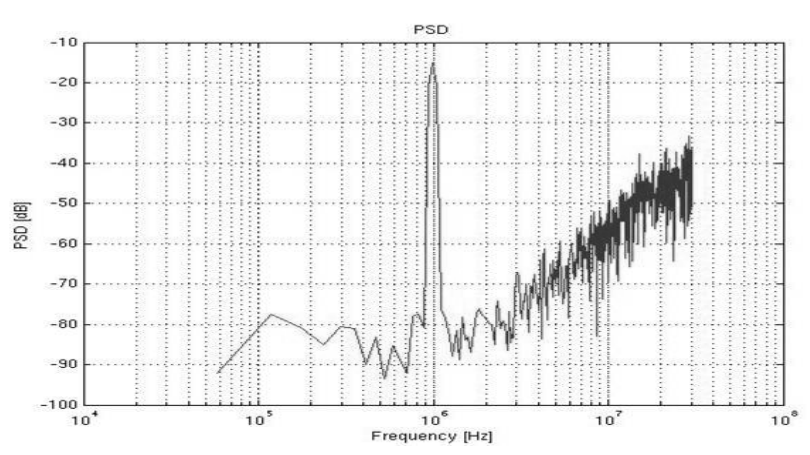

Fig. 7. The power spectral density obtained from Spice simulation.

The quantization noises, $e_{1}(n)$ and $e_{2}(n)$ are equal to the difference between the output voltage of the integrator and the DAC. Therefore, the output voltage of the integrator is sampled on $\mathrm{C}_{\mathrm{Q} 1}$ during $\phi_{1}$ and on $\mathrm{C}_{\mathrm{Q} 2}$ during $\phi_{2}$, for the $1^{\text {st }}$ and $2^{\text {nd }}$ path, respectively. At the following phases of the clocks, $\phi_{1}$ for the $1^{\text {st }}$ path and $\phi_{2}$ for the $2^{\text {nd }}$ path, these capacitors are connected between the input of the opamp and the appropriate DAC voltage in order to integrate the cross-coupled quantization noise on the integrating capacitors $\mathrm{C}_{\mathrm{F} 1}$ and $\mathrm{C}_{\mathrm{F} 2}$. The capacitances of these capacitors are twice of the integrating capacitors $\mathrm{C}_{\mathrm{F} 1}$ and $\mathrm{C}_{\mathrm{F} 2}$. Therefore a gain of two is achieved for the cross-coupling quantization noise. The input sampling capacitors, $\mathrm{C}_{\mathrm{S} 1}$ and $\mathrm{C}_{\mathrm{S} 1}$ are also used for the feedback DAC. The parameters, given in Table I, are also used for the transistor level simulations, except the input signal is chosen to have a frequency of $1 \mathrm{MHz}$ and amplitude of $-12 \mathrm{~dB}$.

The power spectral density of the proposed ADC from Spice simulation is presented in Fig. 7. The noise shaping is $2^{\text {nd }}$ order and the SNDR obtained from Spice simulation is $53 \mathrm{~dB}$ for $2 \mathrm{MHz}$ signal bandwidth. This SNDR value closely matches with the result obtained from Simulink simulations for the same input signal level of $-12 \mathrm{~dB}$, given in Table II. The ADC consumes only $1.56 \mathrm{~mW}$ from a $1.2 \mathrm{~V}$ power supply and provides 9-bit performance for -6dB input level. This corresponds to a figure of merit of $0.75 \mathrm{pJ}$, as defined in [21].

\section{CONCLUSIONS}

Time-interleaved sigma delta ADC architecture with crosscoupled quantization noise is presented. This architecture is composed of $1^{\text {st }}$ order modulators, but the order of the noise shaping is raised to $2^{\text {nd }}$ order by cross-coupling of the quantization noise between stages. Since time-interleaving is used, the $1^{\text {st }}$ order modulators run at half of the output data rate. Therefore, the design constraints on the analog circuitries are relaxed. This enables low power sigma delta ADC designs for the low OSR and wide bandwidth applications. Compared to a traditional $2^{\text {nd }}$ modulator, the proposed sigma delta modulator offers further power and area saving by allowing implementations with just one opamp. The proposed architecture is verified trough the spice simulations of the lowpower ADC designed in 90nm CMOS technology.

\section{REFERENCES}

[1] S.R. Norsworthy, R. Schereier, G.C. Temes, "Delta-Sigma Converters: Theory, Design and Simulation," John Wiley \& Sons, New Jersey, 1997.

[2] W.C. Black, and D.A Hodges, "Time interleaved converter arrays," IEEE J. Solid-State Circuits, vol. 15, no. 6, pp. 1022-1029 Dec 1980.

[3] P. Aziz, H. Sorensen, and J. Van der Spiegel, "Multi-band sigma delta modulation," IEE Electronics Letters, pp. 1673-1674, Sep 1993.

[4] R. F. Cormier Jr, T. L. Sculley, and R. H. Bamberger, "Combining subband decomposition and sigma-delta modulation for wideband A/D conversion," IEEE Proc. Int. Sym. Circuits and Systems, pp. 5.357360, May 1994.

[5] E. King, F. Aram, T.S. Fiez, and I. Galton, "Parallel delta-sigma A/D conversion," IEEE Proc. Custom Integrated Circuits Conf., pp. 503506, May 1994.

[6] H.T. Jensen, and I. Galton, "A robust parallel delta-sigma A/D converter architecture" IEEE Proc. Int. Sym. Circuits and Systems, vol. 2, pp. 1340 - 1343, May 1995

[7] I. Galton, and H.T. Jensen, "Delta-Sigma modulator based A/D conversion without oversampling," IEEE Trans. Circuits and Systems II, vol. 42, pp. 773-784, Dec 1995.

[8] I. Galton, and H.T. Jensen, "Oversampling parallel delta-sigma modulator A/D conversion," IEEE Trans. Circuits and Systems II, vol. 43. no. 12, pp. 801-810, Dec 1996.

[9] E.T. King, A. Eshraghi, and T. Fiez, "A Nyquist-rate delta-sigma A/D converter," IEEE J. Solid-State Circuits, vol. 33, no. 1,pp. 45-52, Jan 1998.

[10] R. Khoini-Poorfard and D. A. Johns, 'Time-interleaved oversampling converters", IEE Electronics Letters, vol. 29,no. 19, pp. 1673-1674, Sep 1993.

[11] R. Khoini-Poorfard and D. A. Johns, "Mismatch effects in timeinterleaved oversampling converters," IEEE Proc. Int. Sym. Circuits and Systems, pp. 5.429-5.432, May 1994.

[12] A. Eshraghi, and T. Fiez, "An area efficient time-interleaved parallel delta-sigma A/D converter," IEEE Proc. Int. Sym. Circuits and Systems, vol. 2, pp. 386-389, May 1998.

[13] M. Kozak and I. Kale, "Novel Topologies for time interleaved deltasigma modulators," IEEE Trans. Circuit and Systems-II, vol. 47, pp. 639-654, Jul 2000.

[14] X. Wang, W. Qin, and X. Ling, "Cascaded parallel oversampling sigma-delta modulators," IEEE Trans. Circuits and Systems II, vol. 47, no. 2, pp. 156-161, Feb 2000.

[15] M. Kozak, M. Karaman and I. Kale, "Efficient architectures for timeinterleaved oversampling delta-sigma converters," IEEE Trans. Circuits and Systems II, vol. 47, no. 8,pp. 802-810, Aug 2000.

[16] K-S. Lee, and F. Maloberti, "Time-interleaved sigma-delta modulator using output prediction scheme," IEEE Trans. Circuits and Systems II, vol. 51, no. 10, pp. 537-541, Oct 2004.

[17] F. Colodro, A. Torralba, and M. Laguna, "Time-interleaved multirate sigma delta modulators," IEEE Int. Sym. Circuits and Systems, vol. 6, pp. 5581-5584, May 2005.

[18] B-S. Song, "A fourth-order bandpass delta-sigma modulator with reduced numbers of op amps," IEEE J. Solid-State Circuits, vol. 30, no. 12, pp. 1309-1315, Dec 1995.

[19] R. Schreier, and G. C. Temes, "Understanding delta-sigma data converters," Wiley \& Sons, New Jersey, 2005.

[20] P. Malcovati, et al., "Behavioral modeling of switched-capacitor sigmadelta modulators," IEEE Trans. Circuits and systems II, vol. 50, no. 3, pp. 352-364, Mar 2003

[21] F. Maloberti, "Architectures and design methodologies for very low power and power effective A/D converters," IEEE North-East Work. on Circuits and Systems, pp. 77-80, June 2006. 